\title{
Correction: ${ }^{18} \mathrm{~F}$-FIBT may expand PET for $\beta$-amyloid imaging in neurodegenerative diseases
}

\author{
Timo Grimmer ${ }^{1} \cdot$ Kuangyu Shi $^{2,3} \cdot$ Janine Diehl-Schmid ${ }^{1} \cdot$ Bianca Natale $^{1} \cdot$ Alexander Drzezga $^{4} \cdot$ Stefan Förster $^{2}$. \\ Hans Förstl ${ }^{1} \cdot$ Markus Schwaiger $^{2} \cdot$ Igor Yakushev $^{2} \cdot$ Hans-Jürgen Wester $^{5} \cdot$ Alexander Kurz $^{1}$. \\ Behrooz Hooshyar Yousefi
}

Published online: 21 November 2018

(c) Springer Nature Limited 2018

Correction to: Molecular Psychiatry; https://doi.org/10. 1038/s41380-018-0203-5; published online 17 August 2018.
The author listing has been updated to indicate that Timo Grimmer and Kuangyu Shi are equally contributing authors.

Timo Grimmer

t.grimmer@1rz.tum.de

1 Department of Psychiatry and Psychotherapy, Klinikum rechts der Isar der Technischen Universität München, Ismaninger Str. 22, 81675 Munich, Germany

2 Department of Nuclear Medicine, Klinikum rechts der Isar der Technischen Universität München, Ismaninger Str. 22, 81675 Munich, Germany

3 Department of Nuclear Medicine, University of Bern, Freiburgstr. 10, 3010 Bern, Switzerland

4 Department of Nuclear Medicine, University Hospital of Cologne, Kerpener Straße 62, 50937 Cologne, Germany

5 Pharmaceutical Radiochemistry, Technische Universität München, Walther-Meißner-Str. 3, 85748 Garching, Germany 